\title{
B-cell-specific elements enhance sustained gene expression mediated by self-replicating extrachromosomal vectors
}

\author{
DOMINIQUE ZEHNPFENNIG ${ }^{1,3}$, HELMUT DEISSLER $^{2}$, AXEL POLACK $^{3}$, DANIEL HERR $^{2}$, \\ GEORG W. BORNKAMM ${ }^{3}$ and CHRISTIAN KURZEDER ${ }^{2}$ \\ Departments of ${ }^{1}$ Child and Adolescent Psychiatry/Psychotherapy, and \\ ${ }^{2}$ Obstetrics and Gynaecology, University of Ulm Medical School, 89075 Ulm; \\ ${ }^{3}$ Institute of Molecular Biology and Tumor Genetics, 81377 Munich, Germany
}

Received March 3, 2010; Accepted April 8, 2010

DOI: $10.3892 / \mathrm{mmr} 00000318$

\begin{abstract}
Retroviral vectors have been considered the most promising vehicles for the transfer of therapeutic genes into cells of the hematopoietic system. In clinical studies, however, cases of leukemoid clonogenic expansion of the transduced cells in vivo caused by semirandom integration of the foreign DNA in the genome have changed the focus to other types of vectors. In addition to their superior safety profile, a higher packaging capacity might be an advantage of non-viral vectors in certain applications. Prolonged transgene expression of non-viral vectors can be achieved by inclusion of EpsteinBarr virus (EBV)-derived elements mediating episomal replication and retention. Furthermore, a variety of cis acting elements have been explored in an attempt to enhance gene transfer efficiency. Our study confirmed that prolonged transgene expression can be achieved in B-lymphoid cells with EBV-derived vectors containing the EBV latent gene EBNA-1 and the EBV latent origin of replication oriP. In addition, we demonstrated that the inclusion of enhancer elements of the immunoglobulin $\kappa$ light chain (Ei and E3') associated with its matrix attachment region (MAR) resulted in a 10 -fold increase in transgene expression in B-lymphoid cells. It can be concluded that these elements are generally useful modules for improving the efficiencies of non-viral vectors in the B-lymphoid lineage.
\end{abstract}

\section{Introduction}

Gene therapy as an option for the treatment of monogenetic diseases and cancer depends on highly efficient gene transfer vectors and satisfactorily resolved safety issues. Furthermore, most therapeutic concepts include sustained expression of the therapeutic gene, which is difficult to achieve with vectors that do not integrate into the target cell's genome. Among

Correspondence to: Dr Christian Kurzeder, Universitätsfrauenklinik Ulm, Prittwitzstr. 43, 89075 Ulm, Germany

E-mail: christian.kurzeder@uniklinik-ulm.de

Key words: B-cell, extrachromosomal vector, Epstein-Barr virus the viral vectors used in gene therapeutic approaches, the retrovirus-based types offer the highest transduction efficiencies in hematopoetic stem cells (1). Clinical trials, however, confirmed a substantial risk of mutagenic insertions and subsequent leukemic expansion of the transduced cells, as was observed in two patients who were treated for X-linked severe combined immunodeficiency (2). Compared to viral particles, non-viral gene transfer vectors combine a superior safety profile with a sufficient packaging capacity. Hence, non-viral vectors are capable of accommodating larger genomic sequences including introns and cis acting elements. Substantial gene transfer rates without adverse effects on the functional integrity and ability of hematopoietic stem cells to form clones could be achieved by electroporation to facilitate the uptake of naked DNA $(3,4)$. Considering recent refinements of biotechnological methods, non-viral in vivo gene transfer with high efficiency into various tissues including muscle, brain and liver, seems feasible (5).

Non-viral gene transfer systems also provide an opportunity to maintain the transferred DNA episomally without integration into the host genome. Plasmids carrying the Epstein-Barr virus (EBV) latent gene EBNA-1 and the EBV latent origin of replication oriP persist in transfected human cells as autonomously replicating extrachromosomal genetic units (6). Most of the generated vectors of this type contain the enhancer/promoter element of the CMV immediate early genes to control transgene expression. In an effort to design a vector allowing sustained and strong gene expression in hematopoietic cells of the B-lineage, the enhancer elements of the immunoglobulin $\kappa$ light chain (Ei and E3') with an optional matrix attachment region (MAR) were inserted into an EBNA1/oriP plasmid. The lacZ gene was used as a reporter gene to quantify transgene expression after transfection of cells of the B-lymphoid lineage, which allowed an assessment of the overall effect of the enhancer elements and of the contributions of each component.

\section{Materials and methods}

Cultivation of cells. The lymphoma cell line DG75, obtained from the American Type Culture Collection (Rockville, MD, USA), was cultivated in RPMI (Invitrogen) supplemented with 


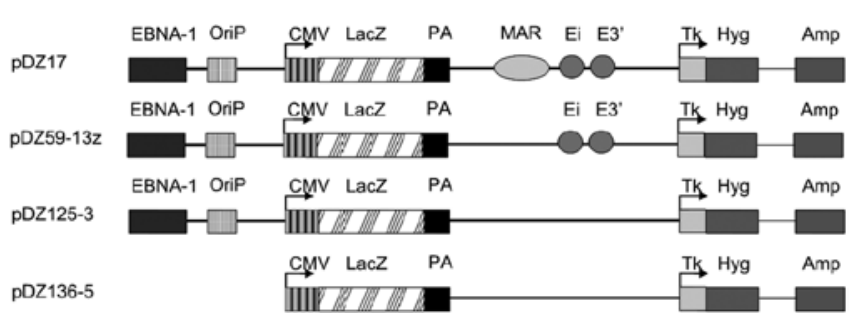

Figure 1. Maps of the EBV-based episomal plasmid vectors pDZ17, pDZ59-13, pDZ125-3 and the control vector pDZ136-5.

$10 \%$ fetal calf serum (Invitrogen), $2 \mathrm{nM}$ L-glutamin (Invitrogen) and $1 \%$ of $10,000 \mu / \mathrm{ml}$ penicillin $/ 10 \mathrm{mg} / \mathrm{ml} \mathrm{streptomycin}$ (Invitrogen) at $37^{\circ} \mathrm{C}$ in an atmosphere containing $5 \% \mathrm{CO}_{2}$.

Construction of modified expression vectors. In order to construct pDZ17 and pDZ59-13, the coding sequence for lacZ was cloned into the SFI-1 site of BC219 and BC229 (7), respectively. The mutant EBV plasmid pDZ59-13 was obtained by cloning the coding sequence for lacZ into the SFI-1 site of BC229. The mutant EBV plasmid pDZ125-3 was generated by HIND-3 digestion of pDZ17 and re-ligation of the large fragment.

Transduction of DG75 cells. DG75 cells were transfected by electroporation: $1 \times 10^{7}$ cells and $20 \mu \mathrm{g}$ DNA were diluted to a total volume of $200 \mu \mathrm{l}$ with Cytomix [120 mM KCl, $0.15 \mathrm{mM}$ $\mathrm{CaCl}_{2}, 10 \mathrm{mM} \mathrm{KH}_{2} \mathrm{PO}_{4}, 25 \mathrm{mM}$ HEPES, 2 mM EGTA, 5 mM $\mathrm{MgCl}_{2}, 2 \mathrm{mM}$ ATP, $5 \mathrm{mM}$ glutathione ( $\mathrm{pH}$ 7.6)] and placed in an electroporation chamber with an electrode distance of $4 \mathrm{~mm}$ to receive a high voltage pulse $(250 \mathrm{~V}$ at a capacitance of $960 \mu \mathrm{F}$ ) in an electroporator (Gene Pulser; Bio-Rad, Munich). After $5 \mathrm{~min}$ on ice, the cells were added to $10 \mathrm{ml}$ of medium and cultivated under standard conditions before being harvested at different time points to measure transgene expression.

$\beta$-galactosidase assay. Cells were washed in phosphatebuffered saline (PBS) and lysed at a density of 105/100 $\mu \mathrm{l}$ in extraction buffer $[100 \mathrm{mM}$ potassium phosphate ( $\mathrm{pH} 7.8$ ), $1 \mathrm{mM}$ DTT, $1 \%$ Triton X-100]. After 5 min of incubation at room temperature, cellular debris was separated by centrifugation at $>10,000 \times \mathrm{g}$. A $10-\mu$ l aliquot of the supernatant was then mixed with $150 \mu \mathrm{l}$ of substrate buffer containing $10 \mu \mathrm{g} / \mathrm{ml}$ of the substrate AMPDG (3-(4-methosyspiro[1,2-dioxetane-3,2'-tricyclo[3.3.1.1.]decan]-4-yl)phenyl- $\beta$ Dgalactopyranosid) (Serva, Heidelberg) in $150 \mu \mathrm{l}$ of $100 \mathrm{mM} \mathrm{Na}_{2} \mathrm{HPO}_{4}(\mathrm{pH} 8.0$ ) and $0.1 \mathrm{mM} \mathrm{MgCl}$. The reaction mixture was incubated for $30 \mathrm{~min}$ at $25^{\circ} \mathrm{C}$, and the reaction was stopped by the addition of $100 \mu \mathrm{l}$ of $0.2 \mathrm{mM} \mathrm{NaOH}$ and $10 \%$ Emerald enhancer (Serva). The absorbance of the product of the enzyme reaction was measured at a wavelength of $420 \mathrm{~nm}$ with a spectrophotometer, and the enzyme activity in relation to the amount of protein in the assay was calculated.

\section{Results}

Biphasic regulation of transgene expression modulated by enhancer elements of the immunoglobin $\kappa$ light chain. The

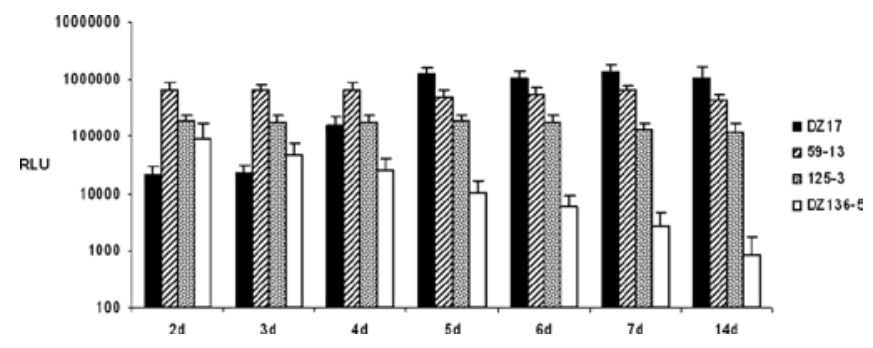

Figure 2. Time course of transgene expression after transduction with EBVbased episomal plasmid vectors. DG-75 cells were transduced with pDZ17, pDZ59-13, pDZ125-3, and the control vector pDZ136-5 and $\beta$ galactosidase expression was measured over a period of 14 days. Transgene expression of pDZ136-5 lacking the EBV viral elements EBNA-1/oriP steadily declined. In contrast, expression mediated by the vectors pDZ125-3 and pDZ50-13 was constant and consistently enhanced. In comparison with pDZ136-5, transgene expression achieved with pDZ17 was increased $\sim 10$-fold. Initially, the MAR element significantly suppressed transgene expression. Means and standard deviation of triplicate experiments are shown

plasmid pDZ17 contains the EBV elements EBNA-1 and oriP, which act together to replicate the viral genome and retain it in the nucleus. Expression of the lacZ transgene is driven by a CMV promoter, and the immunoglobin $(\mathrm{Ig}) \kappa$ light chain MAR as well as its enhancers Ei and E3' are included in the expression cassette (Fig. 1). To examine the effect of the Ig $\kappa$ enhancer cassette, mutants lacking the MAR sequence or the complete Ig $\kappa$ enhancer cassette (MAR, Ei and E3') were generated. The plasmid pDZ136-5 was used to confirm the function of the EBV-derived sequences in a context without the Ig $\kappa$ enhancer cassette. The lymphoma cell line DG-75 was transfected with the EBNA-1/oriP-containing vectors, and transgene expression was measured as enzyme activity over a period of two weeks. Whereas transgene expression from the control vector pDZ136-5 steadily declined and soon reached the background level, transfection with pDZ125-3, which contained the EBV-derived sequences EBNA-1/oriP responsible for episomal replication and retention, resulted in stable transgene expression at high levels over the entire period of observation. Insertion of the immunoglobulin light chain enhancers Ei and E3' (pDZ59-13) led to a substantial increase in transgene expression which was consistently above the level achieved with the EBNA-1/oriP vector pDZ125-3, as shown in Fig. 2. Inclusion of the $\kappa$ light chain MAR element in the expression cassette resulted in an initially suppressed transgene expression before synergistic effects with Ei and E3' increased its level to values even higher than those achieved with pDZ59-13. Compared to the control vector pDZ136-5, transgene expression mediated by pDZ59-13 was approximately 10 -fold higher after the initial lack phase.

Sustained transgene expression in vitro. To examine whether prolonged transgene expression can be achieved with EBNA-1/ oriP vectors carrying the $\mathrm{Ig} \kappa$ enhancer, the time course of transgene expression after transduction of the DG-75 cells was studied. As shown in Fig. 3A, transgene expression from the vector pDZ17 decreased slowly but was maintained over a period of 130 days. On day 10, the level of expression was still $97 \%$ of the maximum value. Transgene expression from the control vector lacking EBNA-1/oriP had already diminished dramatically after 10 days. As shown in Fig. 3B, transgene 
A

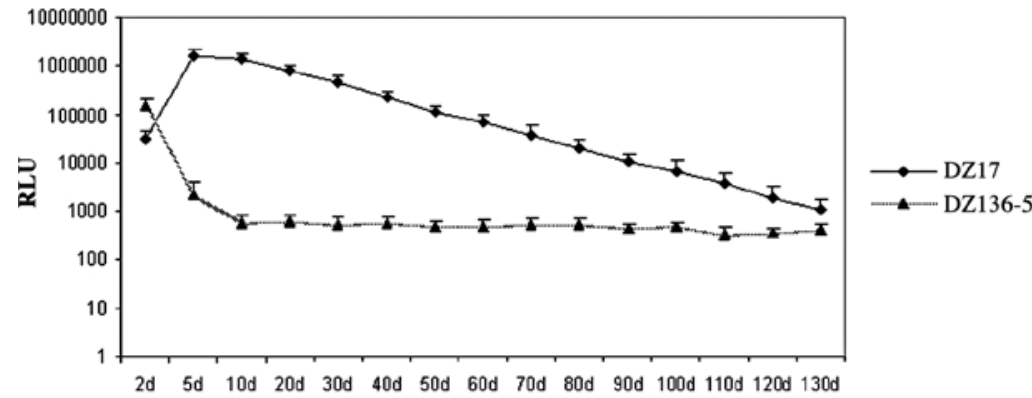

B

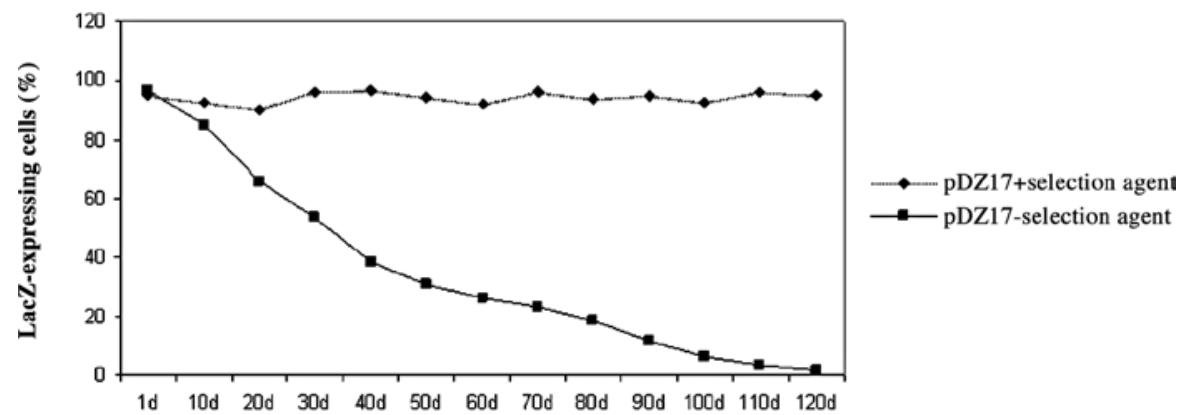

Figure 3. (A) Transgene expression monitored after transduction of the cell line DG-75 with the EBNA-1/oriP vector pDZ17 and the control plasmid pDZ136-5 lacking both the Epstein-Barr viral elements and the Ig $\kappa$ enhancer cassette. (B) Time course of transgene expression after transduction of the cell line DG-75 with the EBNA-1/oriP vector pDZ17 when cultivated with and without Hygromycin, the selection agent.

expression was constant when the cells were cultivated under selection pressure. After removal of the antibiotic, transgene expression declined to reach the detection limit after approximately 130 days.

\section{Discussion}

Sustained transgene expression is essential for the effective and enduring gene therapy of monogenetic diseases. B-lineage-specific transfer and expression of therapeutic genes are considered desirable in cases of genetic diseases solely affecting cells of B-cell origin. To date, recombinant viral vectors have been used almost exclusively in gene transfer studies targeting hematopoietic cells. Retroviral vectors which have already been used in several clinical studies ensure their maintainance in cells through integration into the host cell's chromosomes. However, general considerations regarding safety issues have led to a re-appraisal of non-viral vectors in recent years. Self-replicating EBV-derived episomal vectors were shown to be maintained as an episome in the target cell over a period of 3 months (8). Using an EBNA-1/oriPcontaining vector in combination with in vivo electroporation of muscle cells, prolonged expression of human-FVIII was achieved in animals (9). Our in vitro data support the hypothesis of episomal replication and retention of EBNA-1/oriP vectors by demonstrating long-lasting transgene expression in an EBV-negative cell line.

In an effort to intensify transgene expression in B-lymphoid cells, regulatory elements derived from the immunoglobulin locus have been included in viral and non-viral vectors. As the immunoglobulin heavy chain locus, the $\operatorname{Ig} \kappa$ light chain locus contains multiple enhancers including the MAR/Ei/E3 elements, which play important roles in the regulation of $\mathrm{Ig}$ $\kappa$ transcription and somatic hypermutation (10). Sequences termed MAR that are thought to bind to the nuclear matrix of genomic DNA and facilitate local access of transcription factors to the enhancer sequences within the domain (11) were identified in experiments including DNA binding assays. Although it was an early observation that deletion of the intronic MAR in plasmacytoma cells led to a drastic decrease in gene expression (12), the exact mechanism underlying the modulation of Ig gene expression by MAR is still unclear.

In this study, we demonstrated for the first time that levels of transgene expression mediated by EBNA-1/oriP vectors carrying the $\operatorname{Ig} \kappa$ enhancer cassette are substantially higher in B-lymphoid cells than those achieved with a similar vector lacking the EBV and enhancer elements. These results are in accordance with the observation that lentiviral vectors supplemented with the immunoglobulin-heavy chain enhancer $\mathrm{E} \mu$ with associated MARs are superior to their parental plasmids (13). Transduction with such vectors resulted in enhanced transgene expression in B-lymphocytic cell lines and in human B-lymphocytes differentiated from transduced CD34 ${ }^{+}$ cord blood cells in vitro. Enhanced gene expression was also confined to cells of the B-lymphocytic lineage, whereas insertion of MAR and enhancer elements had no significant effect in cells of other origins, for example T-lymphoid, myeloid, lymphoblastic or carcinoma cell lines. Inclusion of $\operatorname{Ig} \kappa$ MAR into lentiviral vectors resulted in a significant increase in transgene expression and also in the number of proviral DNA copies compared with vectors without this element in HeLa cells (14). Surprisingly, the MAR element initially decreased transgene expression, whereas the vector carrying solely the $\mathrm{Ig} \kappa$ enhancers resulted in an increased transgene expression immediately after transfection. It is possible that the MAR element binds the cellular components generally 
required for proliferation, replication and gene expression, e.g. topoisomerase, and that shortage of these proteins also slowed down transgene expression through a regulatory loop affecting other components of the transcription complex. However, we clearly demonstrated that the insertion of MAR and the Ei and E3' in non-viral vectors is an attractive option by which to increase the level of long-term transgene expression in cells of the B-lymphocytic lineage, and the effects of these genetic elements were quantified. Our results may aid the design of optimized vectors for therapeutic gene transfer into lymphoma cells.

\section{References}

1. Larochelle A and Dunbar CE: Genetic manipulation of hematopoietic stem cells. Semin Hematol: 257-271, 2004

2. Hacein-Bey-Abina S, von Kalle C, Schmidt M, et al: LMO2associated clonal $\mathrm{T}$ cell proliferation in two patients after gene therapy for SCID-X1. Science 302: 415-419, 2003.

3. Van Tendeloo VF, Willems R, Ponsaerts P, et al: High-level transgene expression in primary human $\mathrm{T}$ lymphocytes and adult bone marrow $\mathrm{CD} 34^{+}$cells via electroporation-mediated gene delivery. Gene Ther 7: 1431-1437, 2000.

4. Weissinger F, Reimer P, Waessa T, et al: Gene transfer in purified human hematopoietic peripheral-blood stem cells by means of electroporation without prestimulation. J Lab Clin Med: 138-149, 2003.

5. Bharali DJ, Klejbor I, Stachowiak EK, et al: Organically modified silica nanoparticles: a nonviral vector for in vivo gene delivery and expression in the brain. Proc Natl Acad Sci USA 32: 11539-11544, 2005.
6. Conese M, Auriche $\mathrm{C}$ and Ascenzioni F: Gene therapy progress and prospects: episomally maintained self-replicating systems. Gene Ther 11: 1735-1741, 2004.

7. Polack A, Feederle R, Klobeck G and Hortnagel K: Regulatory elements in the immunoglobulin kappa locus induce c-myc activation and the promoter shift in Burkitt's lymphoma cells. EMBO J 12: 3913-3920, 1993.

8. Black J and Vos JM: Establishment of an oriP/EBNA1-based episomal vector transcribing human genomic beta-globin in cultured murine fibroblasts. Gene Ther 9: 1447-1454, 2002.

9. Mei B, Chen Y, Chen J, Pan CQ and Murphy JE: Expression of human coagulation factor VIII in a human hybrid cell line, HKB11. Mol Biotechnol 34: 165-178, 2006.

10. Inlay MA, Gao HH, Odegard VH, Lin T, Schatz DG and Xu Y: Roles of the Ig kappa light chain intronic and 3' enhancers in Igk somatic hypermutation. J Immunol 177: 1146-1151, 2006.

11. Renz A and Fackelmayer FO: Purification and molecular cloning of the scaffold attachment factor B (SAF-B), a novel human nuclear protein that specifically binds to S/MAR-DNA. Nucleic Acids Res 24: 843-849, 1996.

12. Blasquez VC, Xu M, Moses SC and Garrard WT: Immunoglobulin kappa gene expression after stable integration. I. Role of the intronic MAR and enhancer in plasmacytoma cells. J Biol Chem 264: 21183-21189, 1989.

13. Lutzko C, Senadheera D, Skelton D, Petersen D and Kohn DB: Lentivirus vectors incorporating the immunoglobulin heavy chain enhancer and matrix attachment regions provide positionindependent expression in B lymphocytes. J Virol 77: 7341-7351, 2003.

14. Park F and Kay MA: Modified HIV-1 based lentiviral vectors have an effect on viral transduction efficiency and gene expression in vitro and in vivo. Mol Ther 4: 164-173, 2001. 